\title{
Hiding Negative of an Image using Steganography Even Odd Algorithm for Security Purposes
}

\author{
Ajay Kumar, Mrs. Manu Bansal \\ Department of Electronics and Communication Engineering,Thapar University,Patiala, India. \\ Assistant Professor,Department of Electronics and CommunicationEngineering,Thapar University,Patiala, \\ India.
}

\begin{abstract}
Hence security of data is required during storage and transmission of data. Image Steganography is best popular techniques now a day. The Image hiding in cover image gives us a choice to hide different planes of image in different planes of cover image. In this paper, Negative of original image is hidden in cover image for security purposes. In our implementation pixels of negative image to be substituted in cover image pixels. In our paper Even Odd algorithm is proposed for hiding image in cover image using existing modified LSB algorithm.

Index Terms: Steganography, Cryptography, RGB, Least Significant bit (LSB), pixel.
\end{abstract}

\section{INTRODUCTION}

The past years have been seen a growing interest in data/image confidentiality to defend against eavesdropping and unauthorized access to digital properties. Internet is the most used and fastest medium for communication but it faces many Security related problems like hacking, copyright, eavesdropping etc. This has led to development of techniques in the field of information security, two of which are mainly Cryptography and Steganography [1].

In Cryptography the information encrypts such a way that only sender and receiver parties decode it, but Cryptography is well known technique now days. On the other hand in Steganography the information doesn't alter and hide in such way that only communication parties can know about its presence. So Steganography is a powerful security tool that provides a high level of security.

Steganography is an art and knowledge of unseen communication. The word Steganography is resulting from the Greek word "stegos" meaning "cover" and "grafia" meaning "writing" defining it as "covered writing" [2]. In image the pixels has some integer value, based upon the brightness or color. In image at each pixels value we can hide information. Depend upon the image size we have large no. pixels value available for data hiding. For example in $1024 * 1024$ image size we have 1048576 pixels available on which we can hide data. In color image each pixel depth is 24 bits ( 8 bit for red, 8 bit for green, 8 bit for blue).so in image steganography large amount of information hide as compared to we take other media. Even hiding the data in different planes of image, extraction of original data for unauthorized person is difficult because it's difficult to unauthorized person in which plane in which part we hide information.

This paper proposes hiding negative of an image in cover image for security purposes using even odd Algorithm. The proposed work such that negative image pixels hide in cover image pixels such a way that it's difficult to decode it. Even if unauthorized person decode it, it get the negative of original image not original image. The secret image pixels are coved in 2 least significant bits, 4 least significant bits of the byte composing the carrier image after breaking the image pixels in even odd pixels and hide one plane even odd pixels into different planes. 


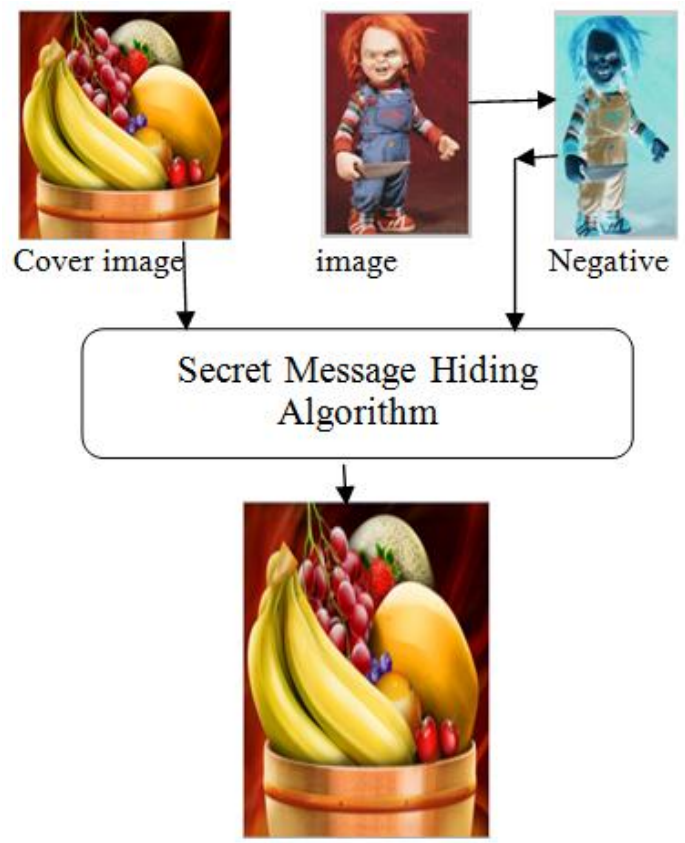

Block Diagram of Image Steganography

\section{Image Steganography Algorithm}

In this paper, for Image hiding is being used existing Image Steganography Algorithm [3, 4].

a) LSB Algorithm

b) Modified LSB Algorithm

Using this Existing algorithm even odd algorithm is proposed.

A. Even Odd Algorithm

a) Read Cover image.

b) Obtain the pixel values of cover image and break the pixels value into three planes red plane, Green Plane, Blue Plane.

c) Choose image for hiding.

d) The hiding image size should be $1 / 8^{\text {th }}$ of the original image in using LSB algorithm.

e) The hiding image size should be $1 / 4^{\text {th }}$ of the original image in using modified LSB algorithm.

f) Obtain the negative of image (which used for hiding) and obtain the pixels value and break into three planes.

g) Break each planes pixel value into even and odd part of pixels.

h) Hide the even odd pixels values in different planes of cover image using existing image steganography algorithm like LSB, modified LSB Algorithm.

i) For hiding the image in cover image xor method is used.

j) After hiding the Stego image is transmitted in communication media.

k) At receiver opposite Retrieved original image after applying Extracting algorithm.

1) In extracting algorithm xoring of original image with Stego image is done to extract the information bits. 


\section{Flow Chart for Even Odd Algorithm}

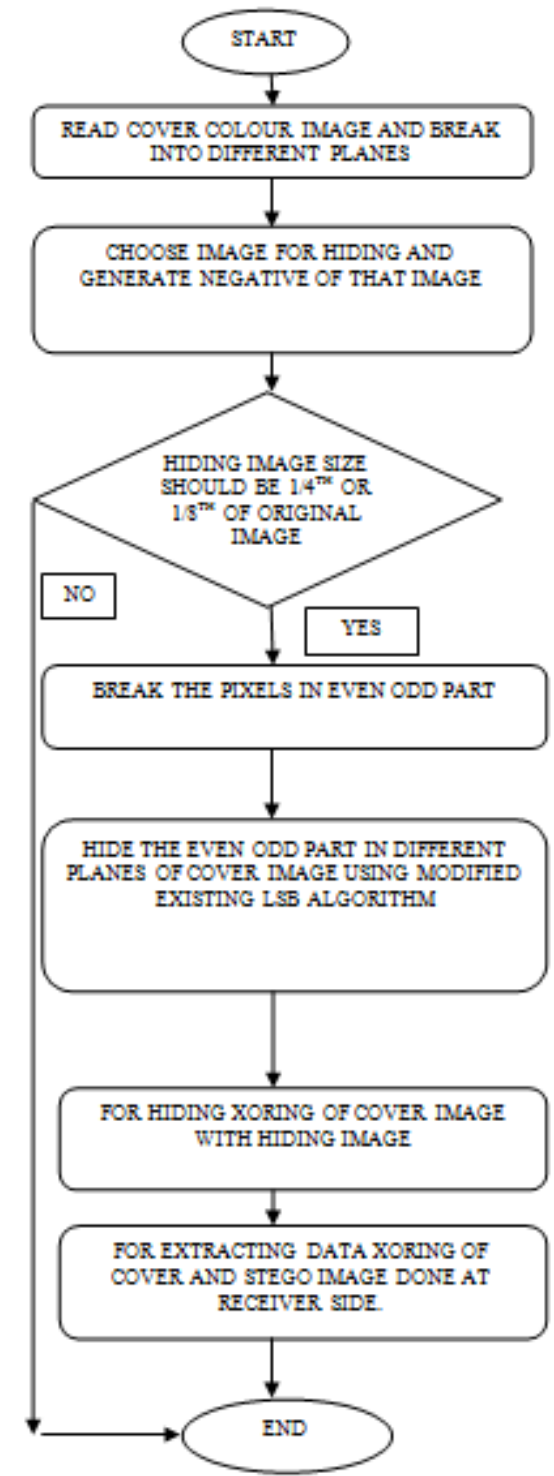

\section{Simulation Resluts:}

In this paper we implement even odd algorithm using existing algorithm in MATLAB 2013. MATLAB, which stands for MATrix LABoratory, is a state-of-the-art mathematical software package, which is used extensively in both academia and industry. It is an interactive program for numerical computation and data visualization, which along with its programming capabilities provides a very useful tool for almost all areas of science and engineering. It is one of the leading software packages for numerical computation. The results of this algorithm as follows:

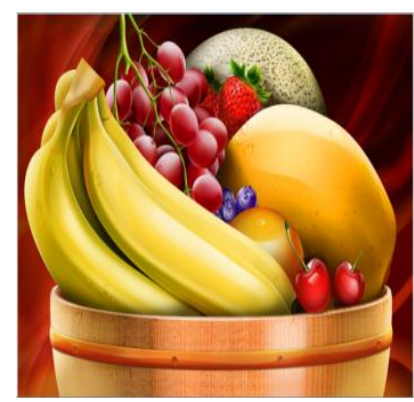

Cover Image 
The hidden Image used in our proposed method is shown below

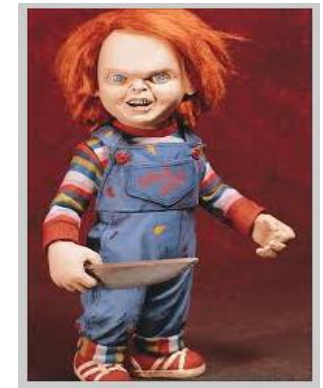

IMAGE

a) The Negative of this image we hide in cover image for security

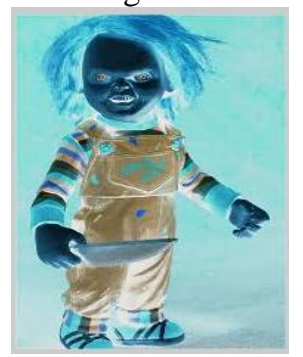

b) Original Cover image is a color image so each pixel has a depth of 24 bits ( 8 bit for red 8 bit for green, 8 bit for blue). So extract the different planes of cover image.

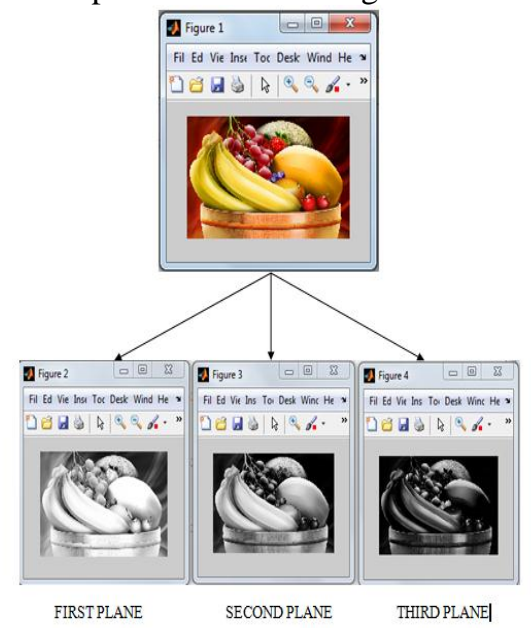

c) Extract the different planes of negative image.




d) Final Stego image after hiding the image in cover image using modified LSB two bits of a byte.

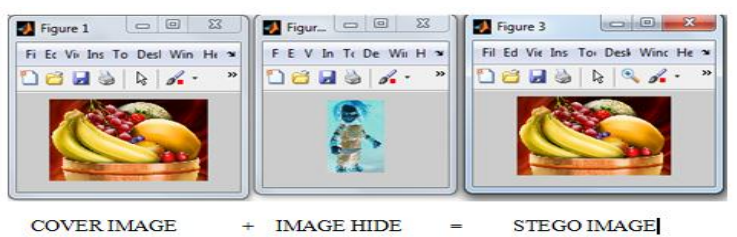

e) Stego image different planes

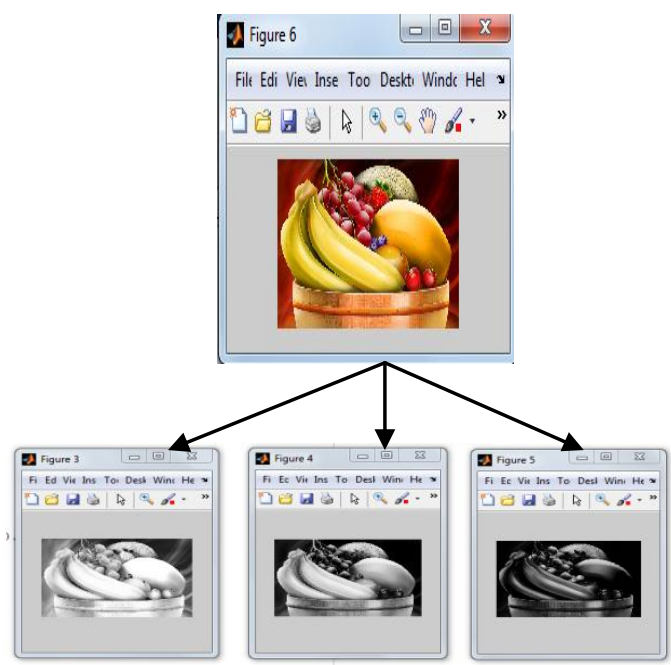

f) Final Stego image after hiding the image in cover image using modified LSB nibble bits of a byte.



COVER IMAGE + IMAGE HIDE = STEGO IMAGEI

g) Final Stego image after hiding the image in cover image using Even Odd algorithm using modified LSB two bits of a byte from LSB.

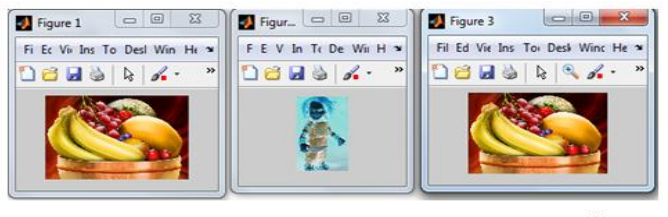

COVER IMAGE + IMAGE HIDE $\quad=$ STEGO IMAGEI

h) Final Stego image after hiding the image in cover image using Even Odd Algorithm using modified LSB nibble bits of a byte.

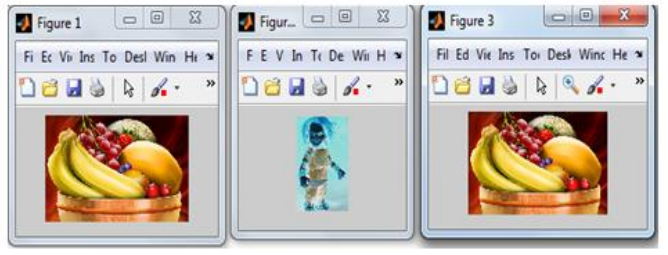

COVER IMAGE + IMAGE HIDE $\quad=$ STEGO IMAGE 


\section{Conclusions}

In this paper Even Odd algorithm is implemented using existing modified LSB of a byte for image hide in a cover image. In our work the negative of original image hide in cover image so it's difficult to extract the original image from cover image because

a) Plane Pixels break into even odd part then hide in cover image.

b) Even if an unknown person extracts the image from cover image they get the negative of original image.

Even generating the negative of original image then hide into cover image using modified LSB algorithm and even odd algorithm the pixels values doesn't vary so much but in even odd algorithm more security of image as compared to modified LSB algorithm.

\section{FUTURE WORK}

As future work, other image Steganography algorithm are to be investigated such as varying the contrast or the gamma level of the carries image, giving the communicating parties more options to parameterize their secret communication.

\section{REFERENCES}

[1] Youssef Bassil "Image Steganography Method based on Brightness Adjustment" Advances in Computer Science and Application (ACSA),ISSN:2166-2924,Vol. 2,No. 2,2012.

[2] Dr. Sudeep D. Thepade,Smita S. Chavan "Cosine, walsh and Slant Wavelet Transforms for Robust Image Steganography" International Conference on wireless and Optical Communication Networks, 26-28 July 2013.

[3] V.Lokeswara Reddy, Dr. A. Subramanyam and Dr. P. Chenna Reddy, "Implementation of LSB Steganography and its evaluation for various file formats" Int. J. Advanced Networking and Application,Vol. 2,pp. 868-872,2011.

[4] Dr. DiwediSamidha, DipeshAgrawal, "Random Image Steganography in Spatial Domain", International Journal of Computer Science and Information Security Publication March 2010, Volume 7 No. 3, March 2013.

[5] Ali K. Hmood, B.B Zaindan "An Overview on hiding information techniques in images" Journal of applied Science 10(18):20942100, 2010 ISSN 1812-5654, 2010 Asian Network for scientific information.

[6] Mohammad TanvirParvez,Adnan Abdul-Aziz Gutub,-“RGB intensity based variable bits image steganography”, IEEE Asia Pacific Services Computing Conference, 2008.

[7] W. Bender, D. Gruhl, N. Morimoto, A. Lu, “Techniques for data hiding IBM systems Journal”, vol.35,no 3,pp. 26-34,1998.

[8] M.M Amin, M. Salleh, S. Lbrahim, M.R.KAtmin, and M.Z.I Shamsuddin, "information hiding using steganography", IEEE $4^{\text {th }}$ National Conference on Telecommunication Technology Proceeding, Shah Alam.Malaysia, pp.21-25,January 2003.

[9] Deshpande Neeta, KamalapurSnehal and Daisy Jacobs, "Implementation of LSB steganography and its evaluation forvarious bits" IEEE $1^{\text {st }}$ International Conference on DigitalInformation Management, India, pp. 173-178, December 2006

[10] J. Madison, S.D. Dickman, “An Overview of Stegano-graphy”, 2007.

[11] A. Gupta and R.Garg, "Detecting LSB Steganographyin Images" 2012. 